\title{
On the nonexistence of minimal strong partial clones
}

\author{
Miguel Couceiro \\ LORIA (CNRS - inria Nancy G.E. - Université de Lorraine) \\ Vandœuvre-lès-Nancy, F-54506, France \\ miguel.couceirodinria.fr \\ Lucien Haddad* \\ Département de Mathématiques et Informatique, Collège Militaire Royal \\ Kingston, Ontario, Canada \\ haddad-1@rmc. ca \\ Karsten Schölzel* \\ dr.karsti@gmail.com
}

* This research was partially conducted while the second and the third authors were visiting LORIA and the Université de Lorraine, in 2016

\begin{abstract}
Let $\mathrm{k}$ be a $k$-element set. We show that the lattice of all strong partial clones on $\mathbf{k}$ has no minimal elements. Moreover, we show that if $C$ is a strong partial clone, then the family of all partial subclones of $C$ is of continuum cardinality. We also show that in almost all cases, every strong partial clone contains a family of continuum cardinality of strong partial subclones.
\end{abstract}

\section{PRELIMINARIES}

In this section we recall basic concepts and terminology needed throughout the paper. For further background, see [2], [4], [5] and [6].

Let $A$ be a finite set with $|A| \geq 2$. If $|A|=k \geq 2$, then we assume that $A=\mathbf{k}:=\{0, \ldots, k-1\}$. For a positive integer $n$, an $n$-ary partial function on $\mathbf{k}$ is a map

$$
f: \operatorname{dom}(f) \rightarrow \mathbf{k}
$$

where $\operatorname{dom}(f)$ is a subset of $\mathbf{k}^{n}$ called the domain of $f$. We denote by $\operatorname{Par}^{(n)}(\mathbf{k})$ the set of all $n$-ary partial functions on $\mathbf{k}$ and we set

$$
\operatorname{Par}(\mathbf{k}):=\bigcup_{n \geq 1} \operatorname{Par}^{(n)}(\mathbf{k})
$$

For positive integers $n, m \geq 1$, and $f \in \operatorname{Par}^{(n)}(\mathbf{k})$ and $g_{1}, \ldots, g_{n} \in \operatorname{Par}^{(m)}(\mathbf{k})$, the composition of $f$ with $g_{1}, \ldots, g_{n}$ is the partial function $f\left[g_{1}, \ldots, g_{n}\right] \in \operatorname{Par}^{(m)}(\mathbf{k})$ whose domain $\operatorname{dom}\left(f\left[g_{1}, \ldots, g_{n}\right]\right)$ is the set of all

$\vec{a} \in \bigcap_{i=1}^{m} \operatorname{dom}\left(g_{i}\right) \quad$ such that $\quad\left(g_{1}(\vec{a}), \ldots, g_{m}(\vec{a})\right) \in \operatorname{dom}(f)$,

and whose values are given by

$$
f\left[g_{1}, \ldots, g_{n}\right](\vec{a}):=f\left(g_{1}(\vec{a}), \ldots, g_{n}(\vec{a})\right)
$$

for all $\vec{a} \in \operatorname{dom}\left(f\left[g_{1}, \ldots, g_{n}\right]\right)$. For every positive integer $n$ and each $1 \leq i \leq n$, we denote by $e_{i}^{n}$ the $n$-ary $i$-th projection function (or, simply, projection) defined by

$$
e_{i}^{n}\left(a_{1}, \ldots, a_{n}\right)=a_{i}
$$

for all $\left(a_{1}, \ldots, a_{n}\right) \in \mathbf{k}^{n}$. Furthermore, we use the notation

$$
J_{\mathbf{k}}:=\left\{e_{i}^{n}: 1 \leq i \leq n, n \in \mathbb{N}\right\}
$$

for the set of all projections.

Definition 1. A partial clone on $\mathbf{k}$ is a subset of $\operatorname{Par}(\mathbf{k})$ closed under composition and containing the set of all projection functions $J_{\mathbf{k}}$.

Remark 1. Partial clones can be equivalently defined in the formalism of Mal'cev; see, e.g., Chapter 20 of [6].

The set of partial clones on $\mathbf{k}$ forms a lattice $\mathcal{L}_{P_{\mathbf{k}}}$ under inclusion, in which the infimum is the set-theoretical intersection. Thus the intersection of arbitrarily many partial clones on $\mathbf{k}$ is itself a partial clone on $\mathbf{k}$. Clearly, the set $J_{\mathbf{k}}$ of all projections on $\mathbf{k}$ is the smallest element in the lattice $\mathcal{L}_{P_{\mathbf{k}}}$ of partial clones.

For $f, g \in \operatorname{Par}^{(n)}(\mathbf{k})$, the partial function $g$ is said to be a subfunction of $f$, fact that we denote by $g \leq f$, if

$$
g=\left.f\right|_{\operatorname{dom}(g)},
$$

i.e., if $\operatorname{dom}(g) \subseteq \operatorname{dom}(f)$ and $g(\vec{a})=f(\vec{a})$ for all $\vec{a} \in$ $\operatorname{dom}(g)$.

Definition 2. A partial clone $C$ on $\mathrm{k}$ is said to be strong if it is closed under taking subfunctions, i.e., if the condition

$$
g \leq f \Longrightarrow g \in C
$$


holds for every $f \in C$ and $g \in \operatorname{Par}(\mathbf{k})$. For $F \subseteq \operatorname{Par}(\mathbf{k})$, we denote its strong closure by $\operatorname{Str}(F)$, i.e.,

$$
\operatorname{Str}(F):=\{g \in \operatorname{Par}(\mathbf{k}): g \leq f \text { for some } f \in F\} .
$$

Example 1. It is easy to see that the set

$$
\{f \in \operatorname{Par}(\mathbf{k}):(0, \ldots, 0) \in \operatorname{dom}(f) \Longrightarrow f(0, \ldots, 0)=0\}
$$

is a strong partial clone on $\mathbf{k}$.

Strong partial clones have been widely studied in the literature, see, e.g., [2], [4], [5] and [6] for a long list of references. The recent paper $[\square]$ is dedicated to the study of strong partial clones of Boolean functions.

It is well known that a partial clone $C$ is strong if and only if $\operatorname{Str}\left(J_{\mathbf{k}}\right) \subseteq C$ (see, e.g., [2], Lemma 2.11). Moreover, it is easy to see that the intersection of an arbitrary family of strong partial clones on $\mathbf{k}$ is also a strong partial clone. In fact, the set of all strong partial clones, ordered by inclusion, also forms a lattice $\mathcal{L}_{\operatorname{Str}\left(P_{\mathbf{k}}\right)}$ whose smallest element is $\operatorname{Str}\left(J_{k}\right)$.

For $F \subseteq \operatorname{Par}(\mathbf{k})$, let pclone $(F)$ denote the strong partial clone generated by $F$, i.e., the intersection of all strong partial clones on $\mathbf{k}$ containing $F$. As it is well-known, strong partial clones coincide exactly with the sets of polymorphisms of relations on $\mathbf{k}$, that we now briefly describe.

For $m \geq 1$, let $\rho$ be an $m$-ary relation on $\mathbf{k}$ (i.e., a subset of $\left.\mathbf{k}^{m}\right)$. Denote by $\mathcal{M}(\rho, \operatorname{dom}(f))$ the set of all $m \times n$ matrices $M$ on $\mathbf{k}$ whose columns $M_{* j} \in \rho$, for $j=1, \ldots, n$ and whose rows $M_{i *} \in \operatorname{dom}(f)$ for $i=1, \ldots, m$. A partial function $f \in \operatorname{Par}^{(n)}(\mathbf{k})$ is said to preserve $\rho \subseteq \mathbf{k}^{m}$ if $\left(f\left(M_{1 *}\right), \ldots, f\left(M_{m *}\right)\right) \in \rho$ for all $M \in \mathcal{M}(\rho, \operatorname{dom}(f))$. Set

$$
\operatorname{pPol} \rho:=\{f \in \operatorname{Par}(\mathbf{k}): f \text { preserves } \rho\} .
$$

It is well known that $\mathrm{pPol} \rho$ is a strong partial clone on $\mathbf{k}$ called the partial clone determined by $\rho$. Note that $f \in \operatorname{pPol} \rho$ if there is no matrix $M$ with columns in $\rho$ and rows in $\operatorname{dom}(f)$.

In this paper we first show that minimal strong partial clones (i.e., atoms of $\mathcal{L}_{\mathrm{Str}\left(P_{\mathrm{k}}\right)}$ ) do not exist. Then we show that the family $\left\{D: D\right.$ is a partial clone and $\left.D \subseteq \operatorname{Str}\left(J_{\mathbf{k}}\right)\right\}$ is of continuum cardinality. Since every strong partial clone contains $\operatorname{Str}\left(J_{\mathbf{k}}\right)$, it follows that any strong partial clone contains a family of partial subclones of continuum cardinality. However, partial clones strictly included in $\operatorname{Str}\left(J_{\mathbf{k}}\right)$ are not strong. In the last section we thus go a bit further and show that, in almost all cases, a strong partial clone contains a family of strong partial subclones of continuum cardinality.

In what follows, by an $n$-ary partial projection we mean a subfunction of a projection function whose domain is a proper subset of $\mathbf{k}^{n}$.

\section{Minimal Strong Partial Clones}

A minimal partial clone on $\mathbf{k}$ is a atom of the lattice $\mathcal{L}_{P_{\mathbf{k}}}$. In other words, a partial clone $C$ is minimal if there is no partial clone $C_{0}$ such that $J_{k} \neq C_{0} \subset C \subset \operatorname{Par}(\mathbf{k})$. Minimal partial clones consisting of partial functions have been completely described in [T], none of which is strong. The natural question is then to ask whether strong minimal partial clones exist, i.e., whether atoms of $\mathcal{L}_{\mathrm{Str}\left(P_{\mathbf{k}}\right)}$ exist.

In this section we show that such partial clones do not exist. This result is not surprising, but to our knowledge it has never been published. The idea behind the proof we present here comes from a discussion between the second author and Ferdinand Börner just after the paper [四] has appeared. We will show that, given a partial function $g$ that is not a partial projection function, we can construct a partial function $h \in \operatorname{pclone}(g)$, with $h \notin \operatorname{Str}\left(J_{\mathbf{k}}\right)$ and such that $g \notin$ pclone $(h)$

Let $g \notin \operatorname{Str}\left(J_{\mathbf{k}}\right)$ be an $n$-ary partial function and $C:=$ pclone $(g)$. We distinguish two cases:

Case 1. For every $\left(a_{1}, \ldots, a_{n}\right) \in \operatorname{dom}(g), g\left(a_{1}, \ldots, a_{n}\right) \in$ $\left\{a_{1}, \ldots, a_{n}\right\}$.

Let $f$ be a restriction of $g$ so that any further restriction of the domain of $f$ results in a partial projection. Let $m:=|\operatorname{dom}(f)|$ and let $\alpha^{1}, \ldots, \alpha^{m} \in \operatorname{dom}(f) \subseteq \mathbf{k}^{n}$ with $\alpha^{i}:=\left(a_{1}^{i}, \ldots, a_{n}^{i}\right)$ and such that

$$
f\left(\begin{array}{ccc}
a_{1}^{1} & \ldots & a_{n}^{1} \\
a_{1}^{2} & \ldots & a_{n}^{2} \\
\vdots & \vdots & \vdots \\
a_{1}^{m} & \ldots & a_{n}^{m}
\end{array}\right)=\left(\begin{array}{c}
b_{1} \\
b_{2} \\
\vdots \\
b_{m}
\end{array}\right) .
$$

Since $f$ is not a partial projection, the right tuple $\left(b_{1}, \ldots, b_{m}\right)^{T}$ is not a column of the matrix on the left. Let $M$ be the $m \times n$ matrix in (II) and let $\rho_{M}$ be the $m$-ary relation on $\mathbf{k}$ consisting of all columns of $M$. Clearly $f \notin \operatorname{pPol}\left(\rho_{M}\right)$, and thus $g \notin \operatorname{pPol}\left(\rho_{M}\right)$.

Let $\bar{f}$ be the $(n+2)$-ary partial function given by

$$
\bar{f}\left(x_{1}, \ldots, x_{n+2}\right):=f\left(x_{1}, \ldots, x_{n}\right)
$$

for all $\left(x_{1}, \ldots, x_{n+2}\right) \in \operatorname{dom}(f) \times \mathbf{k}^{2}$. Note that

$$
\bar{f} \in \operatorname{pclone}(f) \subseteq \operatorname{pclone}(g) .
$$

Now take a $c \in \mathbf{k}, c \neq b_{1}$ and consider the subfunction $h$ of $\bar{f}$ whose domain is the set of all rows of the following matrix:

$$
\left(\begin{array}{ccccc}
a_{1}^{1} & \ldots & a_{n}^{1} & c & b_{1} \\
a_{1}^{1} & \ldots & a_{n}^{1} & b_{1} & c \\
a_{1}^{2} & \ldots & a_{n}^{2} & b_{2} & b_{2} \\
\vdots & & \vdots & \vdots & \vdots \\
a_{1}^{m} & \ldots & a_{n}^{m} & b_{m} & b_{m}
\end{array}\right)
$$

where $b_{1}, b_{2}, \ldots, b_{m}$ are the values of the partial function $f$.

We therefore have

$$
h\left(\begin{array}{ccccc}
a_{1}^{1} & \ldots & a_{n}^{1} & c & b_{1} \\
a_{1}^{1} & \ldots & a_{n}^{1} & b_{1} & c \\
a_{1}^{2} & \ldots & a_{n}^{2} & b_{2} & b_{2} \\
\vdots & & \vdots & \vdots & \vdots \\
a_{1}^{m} & \ldots & a_{n}^{m} & b_{m} & b_{m}
\end{array}\right)=\left(\begin{array}{c}
b_{1} \\
b_{1} \\
b_{2} \\
\vdots \\
b_{m}
\end{array}\right) .
$$


Note that $|\operatorname{dom}(h)|=m+1$.

We show that any restriction of $h$ to a set with at most $m$ tuples is a partial projection. Let $h^{\prime}$ be a restriction of $h$ with $\left|\operatorname{dom}\left(h^{\prime}\right)\right| \leq m$. If $\left(a_{1}^{1}, \ldots, a_{n}^{1}, c, b_{1}\right) \notin \operatorname{dom}\left(h^{\prime}\right)$, then $h^{\prime} \leq e_{n+1}^{n+2}$, i.e., $h^{\prime}$ is a partial projection on its $(n+1)$-st coordinate. Similarly, if $\left(a_{1}^{1}, \ldots, a_{n}^{1}, b_{1}, c\right) \notin \operatorname{dom}\left(h^{\prime}\right)$, then $h^{\prime} \leq e_{n+2}^{n+2}$. On the other hand if $\left(a_{1}^{i}, \ldots, a_{n}^{i}, b_{i}, b_{i}\right) \notin \operatorname{dom}\left(h^{\prime}\right)$ for some $i=2, \ldots, m$, then $h^{\prime}$ is a partial projection function on the same coordinate as the subfunction of $f$ whose domain is contained in the set of rows of $M$ except the $i$-th row.

Now we show that $h \in \operatorname{pPol}\left(\rho_{M}\right)$. Let $N$ be a $m \times(n+2)$ matrix with all columns in $\rho_{M}$ and all rows in $\operatorname{dom}(h)$. Then as $N$ has $m$ rows, the restriction of $h$ on the rows of $N$ is a partial projection function and so it preserves any relation, including the relation $\rho_{M}$. Thus $h \in \operatorname{pPol}\left(\rho_{M}\right)$, i.e., $\operatorname{pclone}(h) \subseteq \operatorname{pPol}\left(\rho_{M}\right)$. Since $g \notin \operatorname{pPol}\left(\rho_{M}\right)$ we conclude that $g \notin \operatorname{pclone}(h)$ and thus pclone $(h) \subsetneq C$.

Case 2. There is an $n$-tuple $\left(a_{1}, \ldots, a_{n}\right) \in \operatorname{dom}(g)$ such that $g\left(a_{1}, \ldots, a_{n}\right) \notin\left\{a_{1}, \ldots, a_{n}\right\}$.

Let $f$ be the restriction of $g$ to the domain $\left\{\left(a_{1}, \ldots, a_{n}\right)\right\}$ and let $f\left(a_{1}, \ldots, a_{n}\right)=b$ with $b \notin\left\{a_{1}, \ldots, a_{n}\right\}$. Choose $c \neq b$ and define the $(n+2)$-ary partial function $h$ by $\operatorname{dom}(h)=\left\{\left(a_{1}, \ldots, a_{n}, c, b\right),\left(a_{1}, \ldots, a_{n}, b, c\right)\right\}$ and $h\left(a_{1}, \ldots, a_{n}, c, b\right)=h\left(a_{1}, \ldots, a_{n}, b, c\right)=b$ and proceed as in Case 1. Thus we have shown the following result.

Theorem 1. Let $C \neq \operatorname{Str}\left(J_{\mathbf{k}}\right)$ be a strong partial clone. Then there is a strong partial clone $C^{\prime}$ such that $\operatorname{Str}\left(J_{\mathbf{k}}\right) \subsetneq C^{\prime} \subsetneq C$. Hence there are no strong minimal partial clones on $\mathbf{k}$.

\section{Clones of Partial Projections}

In this section we consider partial clones consisting only of projections and partial projection functions. For $F \subseteq \operatorname{Par}(\mathbf{k})$, let $\langle F\rangle$ denote the partial clone generated by $F$, i.e., the intersection of all partial clones on $\mathbf{k}$ containing $F$. Notice that $\langle F\rangle$ is not necessarily a strong partial clone.

Let $R_{n}$ denote the set of all $n$-tuples on $\mathrm{k}$ of weight 1 , i.e.,

$$
R_{n}:=\left\{\left(x_{1}, \ldots, x_{n}\right) \in \mathbf{k}^{n}: \sum_{i=1}^{n} x_{i}=1\right\} .
$$

Furthermore, let $p_{n}$ be the $n$-ary partial projection given by

$$
p_{n}\left(x_{1}, \ldots, x_{n}\right):= \begin{cases}x_{1} & \text { if }\left(x_{1}, \ldots, x_{n}\right) \in R_{n} \\ \text { undefined } & \text { otherwise. }\end{cases}
$$

Let $\mathbf{P}:=\left\{p_{n}: n \geq 2\right\}$. We will show that for $t \geq 2$, $p_{t} \notin\left\langle\mathbf{P} \backslash\left\{p_{t}\right\}\right\rangle$. Recall Lemma 2.10 in [2].

Lemma 1. Let $F \subseteq \operatorname{Par}(\mathbf{k})$ and set

$$
\mathcal{D}_{0}:=F \cup J_{\mathbf{k}} \text {. }
$$

For $\ell \geq 0$, define

$$
\begin{aligned}
\mathcal{D}_{\ell+1} & :=\mathcal{D}_{\ell} \cup\left\{f\left[g_{1}, \ldots, g_{n}\right] \mid f \in \mathcal{D}_{0}^{(n)}\right. \text { and } \\
& \left.g_{1}, \ldots, g_{n} \in \mathcal{D}_{\ell}^{(m)} \text { for some } n, m \geq 1\right\} .
\end{aligned}
$$

Then $\langle F\rangle=\bigcup_{\ell \geq 0} \mathcal{D}_{\ell}$.

Fix $t \geq 2$ and $F:=\mathbf{P} \backslash\left\{p_{t}\right\}$. Observe that, since $F \subseteq$ $\operatorname{Str}\left(J_{\mathbf{k}}\right),\langle F\rangle \subseteq \operatorname{Str}\left(J_{\mathbf{k}}\right)$, i.e., $\langle F\rangle$ consists only of projections and partial projections.

Lemma 2. Let $\mathcal{D}_{0}, \mathcal{D}_{1}, \ldots$ be as in Lemma $\mathbb{Z}$. Then, for each $\ell \geq 0, \mathcal{D}_{\ell}$ does not contain any partial projection $p$ of arity $t$ with $R_{t} \subseteq \operatorname{dom}(p)$.

Proof. We prove Lemma $\square$ by induction on $\ell \geq 0$. For $\ell=0$, we have $\mathcal{D}_{0}=\left(\mathbf{P} \backslash\left\{p_{t}\right\}\right) \cup J_{\mathbf{k}}$, which does not contain any partial projection of arity $t$.

Now let $\ell \geq 0$ and suppose that $\mathcal{D}_{\ell}$ does not contain any partial projection $p$ of arity $t$ with $R_{t} \subseteq \operatorname{dom}(p)$. We show that the same holds for $D_{\ell+1}$. By the induction hypothesis and the definition of $D_{\ell+1}$, we need to show that if $f \in \mathcal{D}_{0}^{(n)}$, $n \geq 1, g_{1}, \ldots, g_{n} \in \mathcal{D}_{\ell}^{(t)}$ and $p=f\left[g_{1}, \ldots, g_{n}\right]$, then either $p$ is a projection or a partial projection with $R_{t} \nsubseteq \operatorname{dom}(p)$. Since $g_{1}, \ldots g_{n} \in \mathcal{D}_{\ell}$, it follows from the induction hypothesis that for every $i=1, \ldots, n$, either $g_{i}$ is a total projection or $g_{i}$ is a partial projection with $R_{t} \nsubseteq \operatorname{dom}\left(g_{i}\right)$.

Suppose first that $g_{i}$ is a partial projection for some $i=$

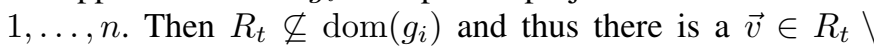
$\operatorname{dom}\left(g_{i}\right)$. But then by the definition of the composition of partial functions $p:=f\left[g_{1}, \ldots, g_{n}\right]$ is not defined on the tuple $\vec{v}$, and thus $\vec{v} \notin \operatorname{dom}(p)$. This shows that $R_{t} \nsubseteq \operatorname{dom}(p)$.

Suppose now that each $g_{1}, \ldots, g_{n}$ is a total projection of arity $t$. If $f \in J_{\mathbf{k}}$ is a projection, then $p=f\left[g_{1}, \ldots, g_{n}\right]$ is a total projection and we are done.

So suppose that $f$ is a partial projection. Since $f \in \mathcal{D}_{0}$, we have $f=p_{n}$ with $n \neq t$.

Case 1. Let $n<t$. Then there is a $j \in\{1, \ldots, t\}$ such $e_{j}^{t} \notin$ $\left\{g_{1}, \ldots, g_{n}\right\}$. Therefore

$$
\vec{v}_{j}:=(0, \ldots, 0, \underbrace{1}_{\mathrm{j}-\text { th position }}, 0, \ldots 0) \notin \operatorname{dom}(p)
$$

since $g_{1}\left(\vec{v}_{j}\right)=\cdots=g_{n}\left(\vec{v}_{j}\right)=0$ and $(0, \ldots, 0) \notin \operatorname{dom}(f)$.

Case 2. Let $t<n$. Then there are $j, k \in\{1, \ldots, t\}$, $j \neq k$, such that $g_{j}=g_{k}$. Without loss of generality, suppose that $g_{j}=g_{k}=e_{j}^{t}$. In this case, the $t$-tuple $\vec{v}_{j}$ defined in (D) does not belong to $\operatorname{dom}(p)$, since the $n$-tuple $\left(g_{1}\left(\vec{v}_{j}\right), \ldots, g_{n}\left(\vec{v}_{j}\right)\right)$ contains at least two entries equal to 1 , and thus $\left(g_{1}\left(\vec{v}_{j}\right), \ldots, g_{n}\left(\vec{v}_{j}\right)\right) \notin \operatorname{dom}(f)$. This completes the proof of Lemma \.

From Lemma 2 , it follows that

$$
p_{t} \notin\left\langle\mathbf{P} \backslash\left\{p_{t}\right\}\right\rangle
$$

for every $t \geq 2$, and thus we have the following corollary.

Corollary 1. Let $X, Y$ be two distinct subsets of $\{2,3, \ldots\}$. Then $\left\langle\left\{p_{i}: i \in X\right\}\right\rangle \neq\left\langle\left\{p_{j}: j \in Y\right\}\right\rangle$.

Let $\mathcal{F}_{J_{\mathbf{k}}}$ be the set of all partial clones contained in $\operatorname{Str}\left(J_{\mathbf{k}}\right)$.

Corollary 2. The set $\mathcal{F}_{J_{\mathbf{k}}}$ is of continuum cardinality on $\mathbf{k}$. 
Now as every strong partial clone on $\mathbf{k}$ contains the set $\operatorname{Str}\left(J_{\mathbf{k}}\right)$ we immediately have the following result.

Theorem 2. Let $C$ be a strong partial clone on $\mathbf{k}$. Then the interval of partial clones $\left[J_{\mathbf{k}}, C\right]$ is of continuum cardinality.

Note that none of the partial clones contained in $\operatorname{Str}\left(J_{\mathbf{k}}\right)$ is strong. Therefore, Theorem $\square$ does not provide any insight into the interval of strong partial clones $\left[\operatorname{Str}\left(J_{\mathbf{k}}\right), C\right]$. Hence, for a strong partial clone $C$, it is natural to ask what is the size of the interval

$\mathcal{C}$ lone $(C):=\{D: D$ is a strong partial subclone of $C\}$.

This is discussed in the next section.

\section{StRong PARTial ClONES GENERATEd By A Single PARTIAL FUNCTION}

Let $f$ be an $n$-ary partial function not in $\operatorname{Str}\left(J_{\mathbf{k}}\right)$. Then there are $m$ tuples $\alpha^{1}, \ldots, \alpha^{m} \in \mathbf{k}^{n}$, with $\alpha^{i}:=\left(a_{1}^{i}, \ldots, a_{n}^{i}\right)$, such that

$$
f\left(\begin{array}{ccc}
a_{1}^{1} & \ldots & a_{n}^{1} \\
\vdots & \vdots & \vdots \\
a_{1}^{i} & \ldots & a_{n}^{i} \\
\vdots & \vdots & \vdots \\
a_{1}^{m} & \ldots & a_{n}^{m}
\end{array}\right)=\left(\begin{array}{c}
b_{1} \\
\vdots \\
b_{i} \\
\vdots \\
b_{m}
\end{array}\right)
$$

and such that $\left(b_{1}, \ldots, b_{m}\right)^{T}$ is not a column of the matrix on the left hand side of the equation. Denote this $m \times n$ matrix by $A$.

Also, throughout this section, assume that the $n$-ary partial function $f$, the matrix $A$ and the number $m$ of rows of $A$ are fixed. Moreover, suppose throughout that the following condition is satisfied:

(*) there is $\alpha \in \mathbf{k}$ such that the $m$-tuple $(\alpha, \ldots, \alpha)^{T}$ is not a column of $A$.

We shall discuss the remaining cases for $f$ in the next section. Now to simplify the notation, we assume $\alpha=0$, i.e., we assume that $(0, \ldots, 0)^{T}$ is not a column of $A$.

Denote by $I_{r}$ the identity matrix of size $r \geq 2$ on $\mathbf{k}$, i.e., $I_{r}$ is the $r \times r$ matrix with symbol 1 on its diagonal and with 0 elsewhere. Furthermore, consider the following infinite set of positive integers

$$
E:=\left\{2 m^{r}+\sum_{1 \leq i \leq r} m^{i-1}: r \geq 0\right\} .
$$

Remark 2. Notice that, for each $r \geq 1$, we have

$2 m^{r}+m^{r-1}+\cdots+m+1=m\left(2 m^{r-1}+\cdots+m+1\right)+1$.

Consequently, if $t>\ell$ with $t, \ell \in E$, then $t \geq m \ell+1$.

We will construct an infinite set of partial functions

$$
\left\{f_{\ell}: \ell \in E\right\}
$$

each of which in pclone $(f)$, and with the property that for every $\ell \in E, f_{\ell} \notin \operatorname{pclone}\left(\left\{f_{k}: k \neq \ell, k \in E\right\}\right)$.

For $\ell \in E$, let $M_{\ell}$ be the matrix whose $n$ first columns are

$$
\left(a_{i}^{1}, \ldots, a_{i}^{1}, a_{i}^{2}, \ldots, a_{i}^{2}, \ldots, a_{i}^{m}, \ldots, a_{i}^{m}\right)^{T}, i=1, \ldots, n
$$

and the $m \times \ell$ last columns are the columns of the identity matrix $I_{m \times \ell}$. Thus, $M_{\ell}$ is a matrix with $m \times \ell$ rows and $n+$ $m \times \ell$ columns. Note also that each of the tuples $\left(a_{1}^{i}, \ldots, a_{n}^{i}\right)$ is repeated $\ell$ times in $M_{\ell}$.

$$
M_{\ell}=\left(\begin{array}{ccc|cccc}
a_{1}^{1} & \ldots \ldots & a_{n}^{1} & 1 & 0 & \ldots \ldots & 0 \\
\vdots & \vdots & \vdots & \vdots & \vdots & \vdots & \\
a_{1}^{1} & \ldots & a_{n}^{1} & 0 & \ldots \ldots & 0 \\
a_{1}^{2} & \ldots & a_{n}^{2} & 0 & \ldots \ldots & 0 \\
\vdots & \vdots & \vdots & \vdots & \vdots & \vdots & \\
a_{1}^{2} & \ldots & a_{n}^{2} & 0 & \ldots \ldots & 0 \\
\vdots & \vdots & \vdots & \vdots & \vdots & \vdots & \\
a_{1}^{m} & \ldots & a_{n}^{m} & 0 & \ldots \ldots & 0 \\
\vdots & \vdots & \vdots & \vdots & \vdots & \vdots & \\
a_{1}^{m} & \ldots & a_{n}^{m} & 0 & \ldots \ldots & 1
\end{array}\right)
$$

Let $\rho_{\ell}$ be the relation that consists of all columns of $M_{\ell}$. Note that $\rho_{\ell}$ is a relation of arity $m \times \ell$ over $\mathbf{k}$.

Now let $f_{\ell}$ be the partial function of arity $n+m \times \ell$ whose domain is the set of all rows of the matrix $M_{\ell}$, and that is given by $f_{\ell}(\vec{v})=f\left(a_{1}^{i}, \ldots, a_{n}^{i}\right)$, where $\left(a_{1}^{i}, \ldots, a_{n}^{i}\right)$ are the first $n$ coordinates of the tuple $\vec{v}$.

Example 2. Let $\mathbf{k}=\{0,1,2\}$, and let $f$ be the ternary partial function defined by

$$
f\left(\begin{array}{lll}
0 & 0 & 1 \\
0 & 1 & 1 \\
2 & 0 & 1
\end{array}\right)=\left(\begin{array}{l}
1 \\
0 \\
0
\end{array}\right)
$$

Here $m=n=3$ and for $7=2 \times 3+1 \in E$, we get

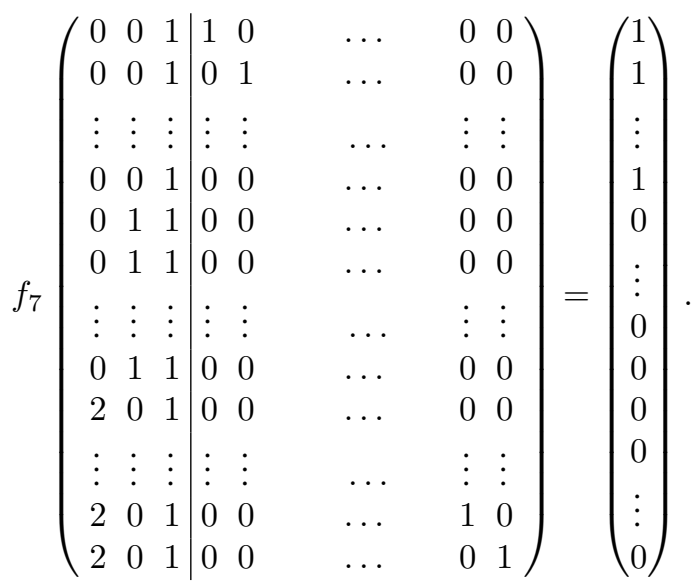


$\underbrace{\underbrace{}_{\text {Note }}}_{I_{21}}$

Note that this matrix has 7 rows starting with $(0,0,1), 7$ rows starting with $(0,1,1)$, and 7 rows starting with $(2,0,1)$.

Lemma 3. For each $\ell \in E$, we have that $f_{\ell} \in \operatorname{pclone}(f)$ and that $f_{\ell}$ is not a partial projection.

Proof. It is easy to see that $f_{\ell} \in \operatorname{pclone}(f)$. Since $f$ is not a partial projection, none of the $n$ first columns of $M_{\ell}$ is equal to $\left(b_{1}, \ldots, b_{1}, b_{2}, \ldots, b_{2}, \ldots, b_{n}, \ldots, b_{n}\right)^{T}$. Moreover, each of the last $m \times \ell$ columns of $M_{\ell}$ consists one 1 and $m \ell-10$ 's, and thus none of those columns can be equal to $\left(b_{1}, \ldots, b_{1}, b_{2}, \ldots, b_{2}, \ldots, b_{n}, \ldots, b_{n}\right)^{T}$.

Lemma 4. If $\ell, t \in E$, then $f_{\ell} \in \operatorname{pPol} \rho_{t}$ if and only if $\ell \neq t$.

Proof. Clearly, $f_{\ell} \notin \mathrm{pPol} \rho_{\ell}$. We show that if $t \neq \ell$, then there is no matrix whose rows are rows of the matrix $M_{\ell}$ and whose columns belong to the relation $\rho_{t}$.

For the sake of contradiction, suppose that such a matrix $B$ exists. Then $B$ has $m \times t$ rows and $n+m \times \ell$ columns. Let $B_{[i, *]}, i=1 \ldots m \times t$, be all the rows of $B$ and $B_{[*, j]}, j=$ $1, \ldots, n+m \times \ell$, be all its columns.

Case 1. $t<\ell$.

For $i=1, \ldots, m \times t$, the $i$-th row of $B$ has the form

$$
B_{[i, *]}=\left(a_{1}^{s}, \ldots, a_{n}^{s}, x_{1}, \ldots, x_{m \times \ell}\right)
$$

where $s \in\{1, \ldots, m\}$ and exactly one of $x_{1}, \ldots, x_{m \times \ell}$ is equal to 1 and all other are 0 . Given that $B$ consists of $m \times t$ such rows and given that $m t<m \times \ell$, there is a $j \in\{n+$ $1, \ldots, n+m \times \ell\}$ such that the column $B_{[*, j]}$ consists of only 0 's. Hence, it does not belong to $\rho_{t}$, which yields the desired contradiction.

Case 2. $\ell<t$.

As $\ell, t \in E$, we must have $t \geq m \times \ell+1$, and

$$
M_{\ell[i, *]}=(a_{1}^{s}, \ldots, a_{n}^{s}, 0, \ldots, 0, \underbrace{1}_{i-\text { th position }}, 0, \ldots, 0)
$$

where $1 \leq s \leq m$. Therefore, if for some $i \in\{1, \ldots, m \times \ell\}$, the $i$-th row $M_{\ell[i, *]}$ of $M_{\ell}$ does not appear in $B$, then $B_{[*, n+i]}$ will contain only 0's, and it will not belong to the relation $\rho_{t}$. Thus every row of $M_{\ell}$ must appear in the matrix $B$. Since $\ell<t$, some of the rows of $M_{\ell}$ are repeated in $B$.

Now if $M_{\ell[i, *]}$ appears repeated exactly $r$ times in $B, 2 \leq$ $r \leq m \times t$, then $B_{[*, n+i]}$ will contain $r$ 1's. Given that this column belongs to $\rho_{t}$ and given that each row of $A$ occurs $t$ times in $M_{t}$, we conclude that $r$ is a multiple of $t$.

For $i=0,1, \ldots, s$ let $r_{i}$ be the number of rows of $M_{\ell}$ that are repeated $i \times t$ times in $B$, with $r_{0}$ being the number of rows in $M_{\ell}$ that appear only once in $B$. Then counting the rows of $B$ we get the equality

$$
r_{0}+t \times r_{1}+2 t \times r_{2}+3 t \times r_{3}+\cdots+s t \times r_{s}=m \times t,
$$

i.e., $r_{0}$ is a multiple of $t$. As $m \times \ell$ is the total number of rows in $M_{\ell}$, and since at least one row of $M_{\ell}$ is repeated in
$B$, we have that $r_{0}<m \times \ell$. Furthermore, as $t \geq m \times \ell+1$, we conclude that $r_{0}<m \times \ell<t$ and thus $r_{0}=0$. Hence, every row of $M_{\ell}$ appears $i \times t$ times in $B$ with $i \geq 1$. It thus follows that $B$ has no column with one 1 and $m \times t-10$ 's, i.e., every column of $B$ is one of the first $n$ columns of $M_{t}$.

Let $B_{R}$ be the block matrix consisting of the last $m \times \ell$ columns to the right of the matrix $B$. Let us count the number of 0 's in $B_{R}$. Since the relation $\rho_{t}$ contains no zero tuple, every column of $B_{R}$ contains at least $t 1$ 's, i.e., every column in $B_{R}$ contains at most $m t-t 0$ 's. Given that there are $m \times \ell$ columns in $B_{R}$, there are at most $m \times \ell \times(m \times t-t) 0$ 's in $B_{R}$. Furthermore, every row of $B$ is a row of $M_{\ell}$, and thus every row of $B_{R}$ contains exactly one 1 and $m \times \ell-10$ 's.

Hence, there are $m \times t \times(m \times \ell-1) 0$ 's in $B_{R}$. Since

$$
m \times t \times(m \times \ell-1)>m \times \ell \times(m \times t-t),
$$

we conclude that at least one column of $B_{R}$ must contain only 0 's, which again yields a contradiction.

Corollary 3. If $X \subseteq E$ and $\ell \notin X$, then $f_{\ell} \notin$ $\operatorname{pclone}\left(\left\{f_{t}: t \in X\right\}\right)$.

Proof. Since $\ell \notin X$, by Lemma 固 we have that $f_{t} \in \operatorname{pPol} \rho_{\ell}$ for every $t \in X$, and thus pclone $\left(\left\{f_{t}: t \in X\right\}\right) \subseteq \operatorname{pPol} \rho_{\ell}$. Since $f_{\ell} \notin \operatorname{pPol} \rho_{\ell}$, we have $f_{\ell} \notin \operatorname{pclone}\left(\left\{f_{t}: t \in X\right\}\right)$.

Now let $C$ be a strong partial clone and suppose that $C$ contains an $n$-ary partial function $f \notin \operatorname{Str}\left(J_{\mathbf{k}}\right)$ such that $|\operatorname{dom}(f)|=m$. Suppose also that $f$ satisfies condition $(*)$. Reconsider the sets $E$ and $\left\{f_{\ell}: \ell \in E\right\}$ defined above. By Corollary 3, for every $X, Y \subseteq E$ with $X \neq Y$, the two strong partial clones $\operatorname{pclone}\left(\left\{f_{t}: t \in X\right\}\right)$ and pclone $\left(\left\{f_{t}: t \in Y\right\}\right)$ are distinct and both are contained in pclone $(f) \subseteq C$. Therefore, we have the following corollary.

Corollary 4. Let $C$ be a strong partial clone on $\mathbf{k}$ and suppose that $C$ contains a partial function $f \notin \operatorname{Str}\left(J_{\mathbf{k}}\right)$ that satisfies $(*)$. Then the set of strong partial clones contained in $C$ is of continuum cardinality.

Example 3. Let $C$ be a strong partial clone on $\mathbf{k}$ and suppose that $C$ contains a unary partial function $g$ such that $1 \in \operatorname{dom}(g)$ and $g(1)=0$. Let $f:=\left.g\right|_{\{1\}}$, i.e., $f$ is given by $\operatorname{dom}(f)=\{1\}$ and $f(1)=0$. Since $C$ is a strong partial clone, we have $f \in C$.

Consider the set $E$ defined above with $m=1$, i.e.,

$$
E=\{2,3, \ldots\} \text {. }
$$

For $\ell \in E$, let $f_{\ell}$ be the $(\ell+1)$-ary partial function whose domain is the set of rows of the matrix

$$
M_{\ell}=\left(\begin{array}{c|ccccc}
1 & 1 & 0 & 0 & \ldots & 0 \\
1 & 0 & 1 & 0 & \ldots & 0 \\
\vdots & \vdots & \vdots & \vdots & \ldots & 0 \\
1 & 0 & 0 & 0 & \ldots & 1
\end{array}\right)
$$

and let $f_{\ell}(\vec{v}):=0$ for all $\vec{v} \in \operatorname{dom}\left(f_{\ell}\right)$. Then $f_{\ell}$ is a 
subfunction of $f\left[e_{1}^{\ell+1}\right]$ and so $f_{\ell} \in C$. By Lemma $\$$ we have that

$$
\operatorname{pclone}\left(\left\{f_{t}: t \in X\right\}\right) \neq \operatorname{pclone}\left(\left\{f_{t}: t \in Y\right\}\right)
$$

for every $X \neq Y \subseteq E$ and so the strong partial clone $C$ contains each member of the family of strong partial clones of continuum cardinality $\left\{\operatorname{pclone}\left(\left\{f_{t}: t \in X\right\}\right): X \subseteq E\right\}$.

\section{FINAL REMARKS}

The construction we presented in Section $\mathbb{\square}$ does not hold if all constant columns belong to the domain. To illustrate this fact, consider the following example.

Example 4. Let $\mathbf{k}=\{0,1,2\}$ and let $f$ be the 4-ary partial function given by

$$
f\left(\begin{array}{llll}
0 & 1 & 2 & 0 \\
0 & 1 & 2 & 1 \\
0 & 1 & 2 & 2
\end{array}\right)=\left(\begin{array}{l}
1 \\
2 \\
0
\end{array}\right)
$$

Note that here $m=3$ and $n=4$. Thus $E=\{2,7,22, \ldots\}$. For $\ell=2$ we have

$$
M_{2}=\left(\begin{array}{llll|llllll}
0 & 1 & 2 & 0 & 1 & 0 & 0 & 0 & 0 & 0 \\
0 & 1 & 2 & 0 & 0 & 1 & 0 & 0 & 0 & 0 \\
0 & 1 & 2 & 1 & 0 & 0 & 1 & 0 & 0 & 0 \\
0 & 1 & 2 & 1 & 0 & 0 & 0 & 1 & 0 & 0 \\
0 & 1 & 2 & 2 & 0 & 0 & 0 & 0 & 1 & 0 \\
0 & 1 & 2 & 2 & 0 & 0 & 0 & 0 & 0 & 1
\end{array}\right)
$$

We want to show that $f_{7} \notin \mathrm{pPol} \rho_{2}$, however the matrix $M_{7}$ associated with the partial function $f_{7}$ is of size $21 \times 25$. So for the sake of brevity we will use $f_{3}$ instead of $f_{7}$. Here the partial function $f_{3}$ is given by

$$
f_{3}\left(\begin{array}{llll|lllllllll}
0 & 1 & 2 & 0 & 1 & 0 & 0 & 0 & 0 & 0 & 0 & 0 & 0 \\
0 & 1 & 2 & 0 & 0 & 1 & 0 & 0 & 0 & 0 & 0 & 0 & 0 \\
0 & 1 & 2 & 0 & 0 & 0 & 1 & 0 & 0 & 0 & 0 & 0 & 0 \\
0 & 1 & 2 & 1 & 0 & 0 & 0 & 1 & 0 & 0 & 0 & 0 & 0 \\
0 & 1 & 2 & 1 & 0 & 0 & 0 & 0 & 1 & 0 & 0 & 0 & 0 \\
0 & 1 & 2 & 1 & 0 & 0 & 0 & 0 & 0 & 1 & 0 & 0 & 0 \\
0 & 1 & 2 & 2 & 0 & 0 & 0 & 0 & 0 & 0 & 1 & 0 & 0 \\
0 & 1 & 2 & 2 & 0 & 0 & 0 & 0 & 0 & 0 & 0 & 1 & 0 \\
0 & 1 & 2 & 2 & 0 & 0 & 0 & 0 & 0 & 0 & 0 & 0 & 1
\end{array}\right)=\left(\begin{array}{l}
1 \\
1 \\
1 \\
2 \\
2 \\
2 \\
0 \\
0 \\
0
\end{array}\right)
$$

Let $\rho_{2}$ be the relation of arity 6 that consists of all columns of $M_{2}$. The matrix below shows that $f_{3} \notin \operatorname{pPol} \rho_{2}$.

$$
f_{3}\left(\begin{array}{llll|lllllllll}
0 & 1 & 2 & 0 & 1 & 0 & 0 & 0 & 0 & 0 & 0 & 0 & 0 \\
0 & 1 & 2 & 0 & 0 & 1 & 0 & 0 & 0 & 0 & 0 & 0 & 0 \\
0 & 1 & 2 & 1 & 0 & 0 & 0 & 1 & 0 & 0 & 0 & 0 & 0 \\
0 & 1 & 2 & 1 & 0 & 0 & 0 & 0 & 1 & 0 & 0 & 0 & 0 \\
0 & 1 & 2 & 2 & 0 & 0 & 0 & 0 & 0 & 0 & 1 & 0 & 0 \\
0 & 1 & 2 & 2 & 0 & 0 & 0 & 0 & 0 & 0 & 0 & 1 & 0
\end{array}\right)=\left(\begin{array}{l}
1 \\
1 \\
2 \\
2 \\
0 \\
0
\end{array}\right)
$$

Therefore our construction is not applicable if we start with an $n$-ary partial function $f$ that has the following property: There are pairwise distinct $i_{0}, i_{1}, \ldots, i_{k-1} \in\{1,2, \ldots, n\}$ such that for every $\vec{v}=\left(v_{1}, \ldots, v_{n}\right) \in \operatorname{dom}(f)$, we have

$$
v_{i_{0}}=0, v_{i_{1}}=1, \ldots, v_{i_{k-1}}=k-1 .
$$

However, we conjecture that Corollary $\mathbb{Q}$ holds for every

$$
C \neq \operatorname{Str}(\mathbf{k}):
$$

Conjecture. Let $C \neq \operatorname{Str}(\mathbf{k})$ be a strong partial clone over $\mathbf{k}$. Then the family of all strong partial clones contained in $C$ is of continuum cardinality.

Acknowledgement. The authors wish to express their many thanks to one of the referees for his / her valuable and constructive remarks.

\section{REFERENCES}

[1] F. Börner, L. Haddad and R. Pöschel, Minimal Partial Clones. Bulletin of the Australian Mathematical Society 44 (1991), pp 405-415.

[2] F. Börner and L. Haddad, Maximal Partial Clones with no finite basis, Algebra Universalis 40 (1998), pp 453-476.

[3] F. Börner, Personal Communications.

[4] M. Couceiro and L. Haddad. A Survey on Intersections of Maximal Partial Clones of Boolean Partial Functions. Proceedings of the 42nd IEEE International Symposium on Multiple-Valued Logic, 2012, Victoria, Canada, pp 287-292.

[5] L. Haddad and K. Schölzel. Countable Intervals of Partial Clones. Proceedings 44th IEEE IEEE International Symposium on MultipleValued Logic, 2014, Bremen, Germany, pp 155-160.

[6] D. Lau, Function algebras on finite sets. A basic course on many-valued logic and clone theory. Springer Monographs in Mathematics (2006).

[7] K. Schölzel. Dichotomy on intervals of strong partial Boolean clones, Algebra Universalis 73 (2015), pp 347-368. 